\title{
Gene Therapy for Pain: Results of a Phase I Clinical Trial
}

\author{
David J. Fink, MD, ${ }^{1}$ James Wechuck, PhD, ${ }^{2}$ Marina Mata, MD, ${ }^{1}$ \\ Joseph C. Glorioso, PhD, ${ }^{3}$ James Goss, PhD, ${ }^{2}$ David Krisky, MD, PhD, ${ }^{2}$ \\ and Darren Wolfe, $\mathrm{PhD}^{2}$
}

Objective: Preclinical evidence indicates that gene transfer to the dorsal root ganglion using replication-defective herpes simplex virus (HSV)-based vectors can reduce pain-related behavior in animal models of pain. This clinical trial was carried out to assess the safety and explore the potential efficacy of this approach in humans.

Methods: We conducted a multicenter, dose-escalation, phase I clinical trial of NP2, a replication-defective HSVbased vector expressing human preproenkephalin (PENK) in subjects with intractable focal pain caused by cancer. NP2 was injected intradermally into the dermatome(s) corresponding to the radicular distribution of pain. The primary outcome was safety. As secondary measures, efficacy of pain relief was assessed using a numeric rating scale (NRS), the Short Form McGill Pain Questionnaire (SF-MPQ), and concurrent opiate usage.

Results: Ten subjects with moderate to severe intractable pain despite treatment with $>200 \mathrm{mg} /$ day of morphine (or equivalent) were enrolled into the study. Treatment was well tolerated with no study agent-related serious adverse events observed at any point in the study. Subjects receiving the low dose of NP2 reported no substantive change in pain. Subjects in the middle- and high-dose cohorts reported pain relief as assessed by NRS and SF-MPQ.

Interpretation: Treatment of intractable pain with NP2 was well tolerated. There were no placebo controls in this relatively small study, but the dose-responsive analgesic effects suggest that NP2 may be effective in reducing pain and warrants further clinical investigation.

ANN NEUROL 2011;70:207-212

A significant limitation to the development of analgesic drugs is that off-target effects at doses below the maximal analgesic threshold restrict the ability to selectively interrupt nociceptive neurotransmission. ${ }^{1}$ To address this limitation, we developed a series of replication-defective herpes simplex virus (HSV)-based vectors to deliver gene expression cassettes directly to dorsal root ganglion (DRG) neurons from skin inoculation. ${ }^{2,3}$ The anatomically defined projection of DRG axons allows targeting of specific ganglia by injection into selected dermatomes. In preclinical studies, the release of antinociceptive peptides or inhibitory neurotransmitters in spinal dorsal horn from the central terminals of transduced DRG neurons effectively reduced pain-related behaviors in rodent models of inflammatory pain, neuropathic pain, and pain caused by cancer. ${ }^{4-9}$
The human PENK gene encodes for preproenkephalin, a precursor protein proteolytically cleaved to produce the endogenous opioid peptides met- and leu-enkephalin. In the spinal cord, enkephalin peptides inhibit pain signaling through actions at presynaptic opioid receptors located on central terminals of primary afferent nociceptors and postsynaptic opioid receptors on second order neurons involved in nociceptive neurotransmission. ${ }^{10} \mathrm{HSV}$ vectors expressing opioid peptides appear to be particularly effective in animal models of inflammatory and cancer pain. ${ }^{4,5,8}$

Because there have been no previous clinical trials of replication-defective HSV as a gene transfer vector, we undertook this phase I dose-escalation study of NP2, a PENK-expressing HSV vector, to assess the safety of the HSV-based gene transfer platform. As secondary outcome

View this article online at wileyonlinelibrary.com. DOI: 10.1002/ana.22446

Received Mar 16, 2011, and in revised form Apr 1, 2011. Accepted for publication Apr 1, 2011.

Address correspondence to Dr Fink, 1500 E Medical Center Drive, Ann Arbor, Ml 48109; E-mail: djfink@umich.edu or Dr Wolfe, 2100 Wharton Street, Suite 701, Pittsburgh, PA 15203. E-mail darren.wolfe@diamyd.com

From the ${ }^{1}$ Department of Neurology, University of Michigan and VA Ann Arbor Healthcare System, Ann Arbor, Ml; ${ }^{2}$ Diamyd, Inc., Pittsburgh, PA; and ${ }^{3}$ Department of Microbiology and Molecular Genetics, University of Pittsburgh, Pittsburgh, PA. 
measures we evaluated the effect of vector-mediated delivery of PENK on pain, and on concurrent use of opiate medication.

\section{Patients and Methods}

\section{Study Design}

The study was sponsored and implemented by Diamyd Incorporated, a subsidiary of Diamyd Medical AB. The protocol was reviewed by the National Institutes of Health Recombinant DNA Advisory Committee, conducted in accordance with the US Food and Drug Administration-approved (investigational new drug \#13613) in compliance with Good Clinical Practice and listed on ClinicalTrials.gov as "Gene transfer for intractable pain: a phase I clinical trial to determine the maximum tolerable dose of a replication-defective herpes simplex virus type I (HSV-1) vector expressing human preproenkephalin (NP2) in patients with malignancies" (\#NCT00804076).

\section{Manufacturing and Preclinical Evaluation}

NP2 is a replication-defective HSV-based vector deleted for the essential immediate early (IE) genes ICP4 and ICP27 with a human cytomegalovirus IE promoter-driven human PENK gene expression cassette inserted into both ICP4 loci. The deletions of the ICP 4 loci also remove the promoters for the ICP22 and ICP 47 genes, rendering NP2 defective in expression of $4 \mathrm{HSV}$ IE genes.

NP2 was propagated in an ICP4 and ICP27 complementing cell line created by sequentially transfecting Vero cells (American Type Culture Collection Catalogue \#CCL-81) with separate plasmids containing ICP4 and ICP27 and drug selection markers. Clonally derived cell lines were tested for complementing activity and stability prior to subsequent retransfection and drug selection. The final isolated cell line, PHC11-54-1, provided stable propagation and amplification of NP2 and was expanded as a master cell bank (MCB). The certified MCB was employed to produce the NP2 master virus bank (MVB) and subsequently to produce the NP2 clinical product in compliance with FDA current Good Manufacturing Practices (cGMP). The MCB, MVB, and clinical cGMP products each passed a thorough panel of safety and identity testing criteria. A Good Laboratory Practice (GLP) preclinical toxicology program was implemented in mice for up to 90 days after inoculation. No target organ of toxicity was identified at any time point. A comprehensive GLP preclinical biodistribution study established that quantifiable vector genomes were limited to the site of injection and the innervating DRG.

The NP2 clinical cGMP stock was produced by infecting 10-layer cell factories of MCB cells with NP2 MVB. The infected culture was harvested, salt treated, and clarified by centrifugation and filtration. Residual DNA was digested with endonuclease, and the vector was purified by ion exchange chromatography. The product was further purified and concentrated by tangential-flow filtration, and recovered NP2 was sterile filtered, formulated, filled into cryovials, and stored below $-70^{\circ} \mathrm{C}$ until use. The final product was tested for safety (including absence of replication competent vector), identity, purity, titer, and enkephalin expression. A stability program was initiated to monitor NP2 product titer, enkephalin expression, $\mathrm{pH}$, appearance, and sterility over the course of the trial.

\section{Subjects}

Subjects 18 years of age or older with intractable pain due to primary or metastatic cancer that was moderate to severe in intensity ( $\geq 40$ on a $100 \mathrm{~mm}$ visual analogue scale) despite (1) stable analgesic treatment with at least $200 \mathrm{mg} /$ day morphine or equivalent or (2) having reached a stable maximum tolerated dose of narcotic or other analgesics that was $<200 \mathrm{mg} /$ day morphine or equivalent were eligible for enrollment.

Trial subjects were excluded if they were pregnant and had to be able to reliably provide pain assessment. Subjects with a clinical diagnosis of active herpetic disease within 6 months of recruitment or vaccination to prevent HSV infection were excluded. We also excluded patients with recent chemotherapy, radiation therapy, or a surgical stabilization procedure. Subjects with serious uncontrolled medical conditions other than malignancy, positive serology for human immunodeficiency virus, hepatitis B or hepatitis C, severe anemia, uncontrolled coagulopathy or bleeding diathesis, or documented immunodeficiency were excluded.

This phase I study was approved by the institutional review boards of the University of Michigan and Chesapeake Research Review and was conducted in accordance with the principles of the Declaration of Helsinki. All subjects executed written informed consent prior to screening. Subjects that passed all inclusion and exclusion screening criteria had vector administered as 10 intradermal injections of approximately $100 \mu \mathrm{l}$ each (total $1.0 \mathrm{ml}$ ) distributed within the dermatome(s) corresponding to the radicular distribution of the pain in a single session on study day 0 . Enrollment of the first cohort commenced with a dose of $1 \times 10^{7}$ plaque forming units (pfu). Subjects were monitored for 4 weeks, and dosing was sequentially escalated to the $1 \times 10^{8}$ and $1 \times 10^{9}$ pfu cohorts following approval by the Data and Safety Monitoring Committee (DSMC).

\section{Study Assessments}

Subjects were observed for at least 12 hours after dosing before being discharged and were scheduled for re-examination at 1 , $3,7,10,14,21$, and 28 days postdosing and monthly thereafter. Safety data examination included adverse events, a full physical examination, vital signs, mucositis evaluation, and clinical laboratory analyses.

Secondary measures included the numeric rating scale (NRS) for pain intensity and the Short Form McGill Pain Questionnaire (SF-MPQ) completed by the subject on study day 0 prior to dosing and on postdosing days 1, 3, 7, 10, 14, 21, and 28. An SF12 short form Health Survey and complete Eastern Cooperative Oncology Group performance status were similarly completed on study day 0 and day 28. Concurrent opiate analgesic usage was recorded on postdosing study days 1 , $3,7,10,14,21$, and 28 . 


\section{Results \\ Subjects}

Ten subjects were enrolled into the study. Four subjects received $1 \times 10^{7}$ pfu NP2, 3 subjects received $1 \times 10^{8}$ pfu NP2, and 3 subjects received $1 \times 10^{9}$ pfu NP2. Subject demographics are presented in Table 1. The mean predosing NRS pain score was $8.0 \pm 0.6$ for subjects in Cohort 1, $9.0 \pm 0.57$ for subjects in Cohort 2, and $7.7 \pm 1.3$ for subjects in Cohort 3 .

\section{Adverse Events}

No treatment-related serious adverse events (SAEs) were reported during the 4-month follow-up period. Of the 10 terminal cancer subjects enrolled, 8 completed the 28-day study, and 4 subjects completed the 4-month follow-up. Four of the 6 discontinued subjects were withdrawn due to death (2 at the low dose, 1 at the mid dose, and 1 in the high-dose cohort); 1 subject requested discontinuation after 105 days of follow-up, and 1 subject discontinued for reasons that were not established. Six of the 10 enrolled subjects reported a total of 8 SAEs. These adverse events included progression of malignancy, obstruction of the common bile duct, pneumonia, asthma, and pleuritic pain. None of the SAEs were judged to be related to the investigational product by the study site principal investigator or the DSMC.

Nine of the 10 enrolled subjects had 1 or more treatment-emergent adverse event (TEAE) during the 4 months following drug delivery. A total of 72 TEAEs were reported. The TEAEs that were deemed as possibly related to treatment were all mild in severity and resolved, including transient injection site erythema and pruritus, and 1 subject who had a transient elevation in body temperature.

No subject seroconverted from anti-HSV antibody (Ab) negative at baseline to $\mathrm{Ab}$ positive at 1 or 4 months after inoculation with NP2, and none of the subjects who were anti-HSV Ab positive at enrollment showed any increase in anti-HSV Ab titer after receiving NP2. No HSV DNA was detected by quantitative polymerase chain reaction (PCR) in the blood or urine of subjects at 1,7 , or 14 days following NP2 dosing.

\section{Secondary Outcomes}

The principal secondary outcome of this trial was the NRS of pain. Because of the terminal nature of subjects with cancer, by 2 months postdosing there was only 1 patient surviving in the $10^{7} \mathrm{pfu}$ cohort, and 2 each in the $10^{8}$ and $10^{9}$ cohorts. By 3 months after dosing, there was only 1 patient remaining in the $10^{7}$ and in the $10^{8}$ cohorts. Therefore, reporting of secondary outcomes is limited to the 8 patients who remained in the study 28 days postdosing.

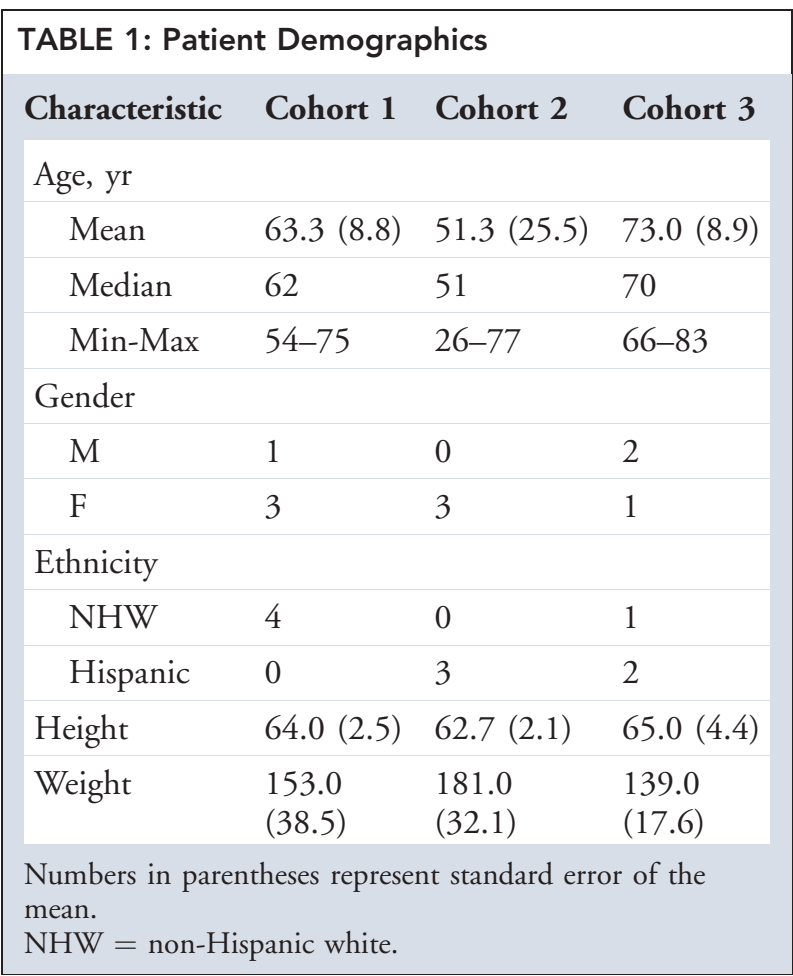

Subjects in the lowest dose cohort $\left(10^{7}\right.$ pfu NP2) did not report substantial changes in NRS over the 28 days following intradermal dosing. Subjects receiving $10^{8}$ pfu NP2 reported an average reduction in NRS pain values at day 1 to approximately $50 \%$ of predosing values. Thereafter, the $10^{8}$ pfu cohort subjects' average NRS values continued to decrease to $<20 \%$ of predosing values at 7 and 14 days, after which the NRS began to rise, although not back to baseline levels. Subjects in the $10^{9}$ pfu NP2 cohort showed a similar reduction in average NRS pain scores to approximately $50 \%$ of baseline at day 1 that continued to decline to approximately $20 \%$ of baseline values by day 7; average NRS pain score remained below this level through the 28-day time point (Fig, A). Although this was not a blinded study, and there were no placebo controls, there was an apparent dose-response that could be measured as the percentage maximal possible effect on the average NRS score from days 7 through 28 (see Fig, B). A similar trend was observed in section I of the subject-reported SF-MPQ scores (Table 2), representing the sum of 15 individual scores (each between 0 and 3) in response to questions about pain (sensory and affective). The dose of concurrent opiate medication that patients continued to take under the direction of their treating physician ranged broadly, and it is difficult to draw conclusions on this parameter given the small number of patients in each cohort (Table 3). 
a

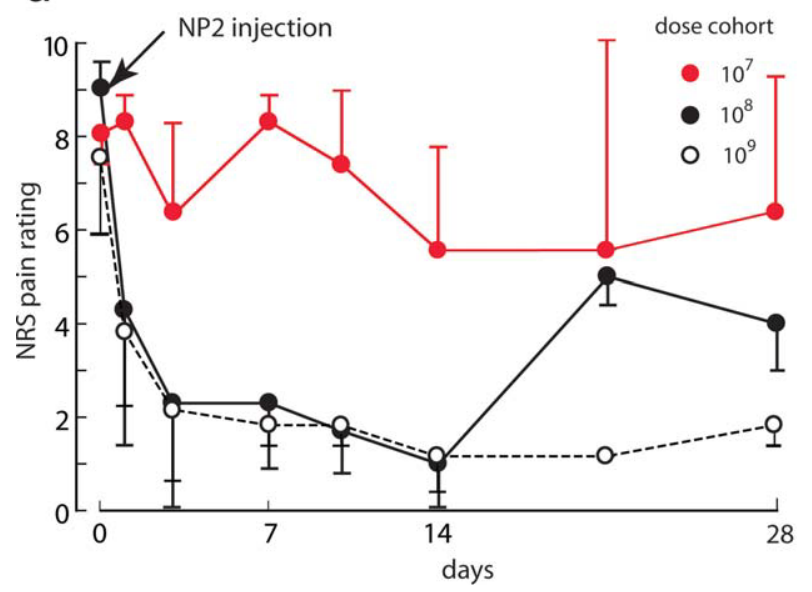

b

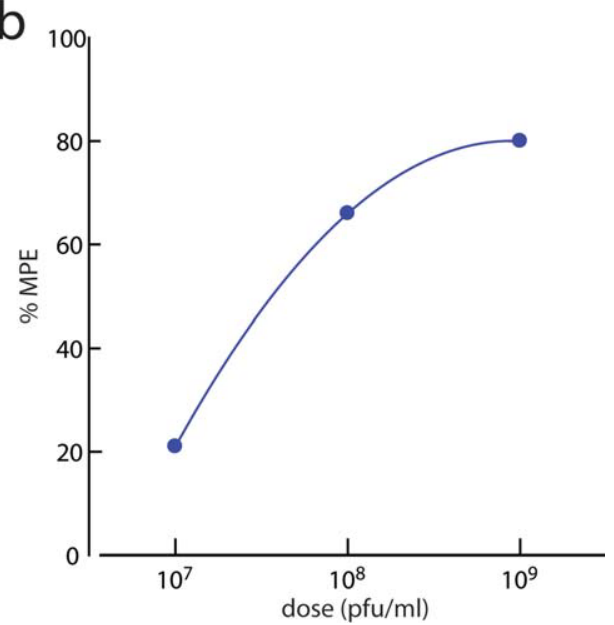

FIGURE : (A) Numeric rating scale (NRS) pain score ( \pm standard error of the mean) for each cohort over the 4 weeks following dosing of NP2. (B) Dose-response curve. \%MPE = percent of maximal potential effect in reducing NRS, calculated using the combined average NRS values at 7, 14, 21, and 28 days after dosing compared to the pretreatment NRS score.

\section{Discussion}

Despite extensive basic research and clinical trials, chronic pain is a condition that often remains refractory to available treatments. ${ }^{11-13}$ Innovative approaches have largely focused on the search for novel targets for analgesic drugs, based on insights gained from broad-based investigations into the neurobiology of chronic pain. ${ }^{14}$ Unfortunately, it has been difficult to identify amenable targets for systemically delivered small molecules that are located exclusively within nociceptive pathways. Not unlike more conventional analgesic agents, ${ }^{15,16}$ even drugs designed to interact with newly identified apparently nociceptive-specific targets such as the vanilloid receptor TRPV1 have been found to have biological roles unrelated to nociception that pose a significant constraint to their use. ${ }^{17,18}$

HSV is a ubiquitous, naturally neurotropic virus spread by contact of skin or mucous membranes that establishes a persistent latent state in neurons of sensory ganglia. By the same cellular transduction mechanisms, replication-defective HSV-based vectors delivered subcutaneously or intradermally transfer genetic material into

\begin{tabular}{|llll|}
\hline \multicolumn{4}{|l|}{ TABLE 2: Short Form McGill Pain Questionnaire } \\
Score and Range of Scores at 1-Week Intervals \\
up to 28 Days after Dosing \\
Day & Cohort 1 & Cohort 2 & Cohort 3 \\
0 & $18(10-32)$ & $32(17-42)$ & $25(18-32)$ \\
7 & $18(7-35)$ & $11(0-22)$ & $6(1-11)$ \\
14 & $17(3-41)$ & $5(0-13)$ & $5(1-9)$ \\
21 & $22(5-39)$ & $13(6-22)$ & $8(1-16)$ \\
28 & $19(9-41)$ & $12(2-15)$ & $1(0-1)$ \\
\hline
\end{tabular}

DRG neurons in vivo. ${ }^{19}$ The human PENK gene carried by NP2 encodes preproenkephalin, a precursor protein that is processed to produce 6 met-enkephalin and 1 leuenkephalin moieties; endogenous opioid peptides that are the naturally occurring high-affinity ligands of the delta opioid receptor. ${ }^{10}$ In this clinical trial, the mechanism of action of the vector was not directly confirmed, but in animal studies the analgesic effects achieved by HSVmediated expression of PENK are blocked by opioid receptor antagonists naloxone and intrathecal naltrexone, ${ }^{4-}$ ${ }^{6}$ suggesting a site of action at spinal opioid receptors, and in vitro biological effects of vector-produced enkephalins are blocked by $10 \mathrm{pM}$ naltrindole ${ }^{20}$ consistent with in vitro activity at the delta opioid receptor. Although met-enkephalin has a higher affinity for the delta compared to the mu opioid receptor, ${ }^{21}$ it is certainly possible that the analgesic effects observed are mediated through mu as well as delta opioid receptors.

Conceptually, HSV-mediated delivery of PENK represents a logical extension of the technique of

TABLE 3: Morphine Equivalent Dose of Opiate Medication Taken by Subjects up to 28 Days after Treatment with NP2 Presented as Mean Dose (Milligrams Morphine Equivalent) and the Range at Each Time Point

\begin{tabular}{|llll}
\hline Day & Cohort 1 & Cohort 2 & Cohort 3 \\
\hline 0 & $182(81-360)$ & $215(15-400)$ & $377(45-708)$ \\
7 & $373(84-930)$ & $115(15-231)$ & $377(45-708)$ \\
14 & $266(63-630)$ & $115(15-231)$ & $399(45-753)$ \\
21 & $267(64-630)$ & $82(0-231)$ & $399(45-753)$ \\
28 & $267(66-630)$ & $115(15-231)$ & $399(45-753)$ \\
\hline
\end{tabular}


delivering opiate drugs by intrathecal infusion ${ }^{22}$ to maximize effects achieved at the spinal level while minimizing systemic side effects. Following delivery, vector particles are taken up by nerve terminals in the skin and transported to the DRG, where the circularized vector genome establishes a persistent state as an intranuclear episomal element. Vector-derived expression of human PENK in DRG following skin inoculation of HSV vectors in rodents has been confirmed by in situ hybridization, reverse transcriptase PCR, radioimmunoassay and immunocytochemistry. ${ }^{4,23}$ Although we were not able to study vector biodistribution in this clinical trial, preclinical studies indicate that replication-defective vector genomes are constrained to the injection site and related DRG. This was confirmed by a preclinical GLP biodistribution study of NP2, in which 16 different tissues were examined between 1 and 91 days after inoculation.

Other gene transfer approaches including intrathecal injection of adenovirus, ${ }^{24}$ adeno-associated virus, ${ }^{25}$ and naked plasmids ${ }^{26}$ have been demonstrated to reduce pain-related behaviors in animal models of pain, but none of these approaches has yet been brought to a human clinical trial. In preclinical animal studies, skin inoculation of HSV vectors expressing PENK have reduced acute hyperalgesic responses, ${ }^{27}$ as well as painrelated behaviors in models of arthritis, ${ }^{28}$ formalin injection, ${ }^{4}$ peripheral nerve damage, ${ }^{6}$ and bone cancer. ${ }^{5}$ Because this was the first human trial employing HSV vectors to achieve gene transfer, we elected to carry out the phase I clinical trial for safety and dose finding in patients with pain caused by cancer.

The safety profile observed in this study was not unanticipated. Oncolytic recombinant HSV-1 viruses without transgenes that are intended to kill malignant cells by limited replication have been injected directly into tumors in brain, liver, and skin in $>200$ subjects to date with no reported test agent-related SAEs. ${ }^{29-33}$ Replication-competent HSV recombinants have also been examined in clinical trials as potential vaccines against genital herpes. Although these approaches have in some cases generated quite high anti-HSV antibody levels (without effectively preventing HSV infection), no drugrelated SAEs have been observed. ${ }^{34-36}$

This phase I clinical trial primarily addressed the question of whether intradermal delivery of NP2 to skin would prove to be safe and well tolerated by subjects. The small number of patients and the absence of placebo controls warrant circumspect interpretation of the secondary outcome measures. But the observation that subjects in the low-dose cohort had little change in the NRS or SF-MPQ whereas subjects in the higher-dose cohorts reported substantial reduction in NRS and improvement in SF-MPQ is encouraging. Based partially upon these results, Diamyd has initiated a randomized, double-blind placebo-control phase II clinical trial in a similar patient population ("A phase II, randomized, double blind, placebo-controlled, multicenter study to investigate the impact of NP2 in subjects with intractable pain due to malignancy"; ClinicalTrials.gov \#NCT01291901).

Although pain associated with cancer is a significant clinical problem with unmet medical need, ${ }^{37}$ the potential utility of HSV-mediated gene transfer to the DRG from skin inoculation is not limited to treatment of cancer pain. The recombinant replication-defective HSV approach represents a platform technology-nerve targeting drug delivery system (NTDDS)-that can be used to deliver and express any of a number of genes in the nervous system. A related NTDDS vector, NG2, which expresses human glutamic acid decarboxylase to effect the release of the inhibitory neurotransmitter $\gamma$ aminobutyric acid, reduces pain-related behaviors in preclinical models of neuropathic pain from nerve injury ${ }^{38}$ and diabetes. ${ }^{39}$ Clinical trials of NTTDS vectors in pain have the advantage that the biological effect of the transgene product can be assessed continuously in real time. NTDDS gene transfer to the DRG to express neurotrophins locally prevents the progression of polyneuropathy in relevant preclinical models, ${ }^{40-42}$ suggesting that the NTDDS platform may be used to treat degenerative polyneuropathies as well.

\section{Acknowledgments}

D.J.F. and M.M. were supported in part by NIH grants NIDDK DK044935 and NINDS NS038850, and received a grant in support of the clinical trial from Diamyd. Patients enrolled at the University of Michigan were supported in part by NCRR UL1RR024986. J.C.G. was supported in part by NIDDK DK044935.

We thank Drs S. Urba, S. Chiravuri, W. Goins, and S. Huang; and K. Weatherwax, G. Estep, H. L'Esperance, M. Orr, S. Amaba; and J. Dunn.

\section{Potential Conflicts of Interest}

D.J.F.: patents, royalties, University of Pittsburgh. J.W.: employment, stock/stock options, Diamyd. J.C.G.: patents, royalties, stock/stock options, Diamyd. J.G.: employment, Diamyd. D.K.: employment, stock/stock options, Diamyd; patents, royalties, University of Pittsburgh. D.W.: board membership, employment, stock/stock options, Diamyd; patents, royalties, University of Pittsburgh. 


\section{References}

1. Mata M, Hao S, Fink DJ. Applications of gene therapy to the treatment of chronic pain. Curr Gene Ther 2008;8:42-48.

2. Wolfe D, Goins WF, Yamada M, et al. Engineering herpes simplex virus vectors for CNS applications. Exp Neurol 1999;159:34-46.

3. Glorioso JC, Fink DJ. Herpes vector-mediated gene transfer in treatment of diseases of the nervous system. Annu Rev Microbiol 2004;58:253-271.

4. Goss JR, Mata M, Goins WF, et al. Antinociceptive effect of a genomic herpes simplex virus-based vector expressing human proenkephalin in rat dorsal root ganglion. Gene Ther 2001;8:551-556.

5. Goss JR, Harley CF, Mata M, et al. Herpes vector-mediated expression of proenkephalin reduces pain-related behavior in a model of bone cancer pain. Ann Neurol 2002;52:662-665.

6. Hao S, Mata M, Goins W, et al. Transgene-mediated enkephalin release enhances the effect of morphine and evades tolerance to produce a sustained antiallodynic effect. Pain 2003;102:135-142.

7. Wolfe D, Hao S, Hu J, et al. Engineering an endomorphin-2 gene for use in neuropathic pain therapy. Pain 2007;133:29-38.

8. Hao S, Wolfe D, Glorioso JC, et al. Effects of transgene-mediated endomorphin-2 in inflammatory pain. Eur J Pain 2009;13:380-386.

9. Liu J, Wolfe D, Hao S, et al. Peripherally delivered glutamic acid decarboxylase gene therapy for spinal cord injury pain. Mol Ther 2004;10:57-66.

10. Dickenson AH, Kieffer B. Opiates: basic mechanisms. In: McMahon SB, Koltzenburg M, eds. Melzack and Wall's textbook of pain. New York, NY: Elsevier, 2006:427-442.

11. Kalso E, Edwards JE, Moore RA, McQuay HJ. Opioids in chronic non-cancer pain: systematic review of efficacy and safety. Pain 2004;112:372-380.

12. Bjordal JM, Klovning A, Ljunggren AE, Slordal L. Short-term efficacy of pharmacotherapeutic interventions in osteoarthritic knee pain: a meta-analysis of randomised placebo-controlled trials. Eur J Pain 2007;11:125-138.

13. Finnerup NB, Sindrup SH, Jensen TS. The evidence for pharmacological treatment of neuropathic pain. Pain 2010;150:573-581.

14. Woolf CJ. Central sensitization: implications for the diagnosis and treatment of pain. Pain 2011;152(3 suppl):S2-S15.

15. Cherny N, Ripamonti C, Pereira J, et al. Strategies to manage the adverse effects of oral morphine: an evidence-based report. J Clin Oncol 2001;19:2542-2554.

16. Tassinari D, Sartori S, Tamburini E, et al. Adverse effects of transdermal opiates treating moderate-severe cancer pain in comparison to long-acting morphine: a meta-analysis and systematic review of the literature. J Palliat Med 2008;11:492-501.

17. Caterina MJ. On the thermoregulatory perils of TRPV1 antagonism. Pain 2008;136:3-4.

18. Gavva NR, Treanor JJ, Garami A, et al. Pharmacological blockade of the vanilloid receptor TRPV1 elicits marked hyperthermia in humans. Pain 2008;136:202-210.

19. Glorioso JC, DeLuca NA, Fink DJ. Development and application of herpes simplex virus vectors for human gene therapy. Annu Rev Microbiol 1995;49:675-710.

20. Chattopadhyay M, Mata M, Fink DJ. Continuous delta opioid receptor activation reduces neuronal voltage gated sodium channel (NaV1.7) levels through activation of protein kinase $C$ in painful diabetic neuropathy. J Neurosci 2008;28:6652-6658.

21. Paterson SJ, Robson LE, Kosterlitz HW. Classification of opioid receptors. Br Med Bull 1983;39:31-36.

22. Wallace $M$, Yaksh TL. Long-term spinal analgesic delivery: a review of the preclinical and clinical literature. Reg Anesth Pain Med 2000;25:117-157.
23. Antunes Bras JM, Epstein AL, Bourgoin $S$, et al. Herpes simplex virus 1-mediated transfer of preproenkephalin $A$ in rat dorsal root ganglia. J Neurochem 1998;70:1299-1303.

24. Finegold AA, Mannes AJ, ladarola MJ. A paracrine paradigm for in vivo gene therapy in the central nervous system: treatment of chronic pain. Hum Gene Ther 1999;10:1251-1257.

25. Storek $B$, Reinhardt $M$, Wang $C$, et al. Sensory neuron targeting by self-complementary AAV8 via lumbar puncture for chronic pain. Proc Natl Acad Sci U S A 2008;105:1055-1060.

26. Milligan ED, Sloane EM, Langer SJ, et al. Repeated intrathecal injections of plasmid DNA encoding interleukin-10 produce prolonged reversal of neuropathic pain. Pain 2006;126:294-308.

27. Wilson SP, Yeomans DC, Bender MA, et al. Antihyperalgesic effects of infection with a preproenkephalin-encoding herpes virus. Proc Natl Acad Sci U S A 1999;96:3211-3216.

28. Braz J, Beaufour C, Coutaux A, et al. Therapeutic efficacy in experimental polyarthritis of viral-driven enkephalin overproduction in sensory neurons. J Neurosci 2001;21:7881-7888.

29. Markert JM, Medlock MD, Rabkin SD, et al. Conditionally replicating herpes simplex virus mutant, G207 for the treatment of malignant glioma: results of a phase I trial. Gene Ther 2000;7:867-874.

30. Rampling R, Cruickshank G, Papanastassiou V, et al. Toxicity evaluation of replication-competent herpes simplex virus (ICP 34.5 null mutant 1716) in patients with recurrent malignant glioma. Gene Ther 2000;7:859-866.

31. Papanastassiou V, Rampling $R$, Fraser $M$, et al. The potential for efficacy of the modified (ICP $34.5(-)$ ) herpes simplex virus HSV1716 following intratumoural injection into human malignant glioma: a proof of principle study. Gene Ther 2002;9:398-406.

32. Harrow S, Papanastassiou V, Harland J, et al. HSV1716 injection into the brain adjacent to tumour following surgical resection of high-grade glioma: safety data and long-term survival. Gene Ther 2004; 11:1648-1658.

33. Nakao A, Kimata $H$, Imai $T$, et al. Intratumoral injection of herpes simplex virus HF10 in recurrent breast cancer. Ann Oncol 2004;15:988-989.

34. de Bruyn G, Vargas-Cortez M, Warren T, et al. A randomized controlled trial of a replication defective ( $\mathrm{gH}$ deletion) herpes simplex virus vaccine for the treatment of recurrent genital herpes among immunocompetent subjects. Vaccine 2006;24:914-920.

35. Casanova G, Cancela R, Alonzo L, et al. A double-blind study of the efficacy and safety of the ICP10deltaPK vaccine against recurrent genital HSV-2 infections. Cutis 2002;70:235-239.

36. Jones CA, Cunningham AL. Vaccination strategies to prevent genital herpes and neonatal herpes simplex virus (HSV) disease. Herpes 2004;11:12-17.

37. Cleeland CS, Gonin R, Hatfield AK, et al. Pain and its treatment in outpatients with metastatic cancer. N Engl J Med 1994;330: 592-596.

38. Hao S, Mata M, Wolfe D, et al. Gene transfer of glutamic acid decarboxylase reduces neuropathic pain. Ann Neurol 2005;57:914-918.

39. Chattopadhyay M, Mata M, Fink DJ. Vector-mediated release of GABA attenuates pain-related behaviors and reduces $\mathrm{Na}(\mathrm{V}) 1.7$ in DRG neurons. European Journal of Pain 2011; epub ahead of print. PMID 21488703.

40. Chattopadhyay M, Mata M, Goss J, et al. Prolonged preservation of nerve function in diabetic neuropathy in mice by herpes simplex virus-mediated gene transfer. Diabetologia 2007;50:1550-1558.

41. Chattopadhyay M, Goss J, Wolfe D, et al. Protective effect of herpes simplex virus-mediated neurotrophin gene transfer in cisplatin neuropathy. Brain 2004;127:929-939.

42. Chattopadhyay $M$, Wolfe $D$, Huang $S$, et al. In vivo gene therapy for pyridoxine-induced neuropathy by herpes simplex virus-mediated gene transfer of neurotrophin-3. Ann Neurol 2002;51:19-27. 